\title{
Reprogrammability and Scalability of Magnonic Fibonacci Quasicrystals
}

\author{
Filip Lisiecki, ${ }^{1,}$ Justyna Rychły, ${ }^{2}$ Piotr Kuświk, ${ }^{1}$ Hubert Głowiński, ${ }^{1}$ Jarosław W. Kłos, ${ }^{2,3}$ \\ Felix Groß, ${ }^{4}$ Iuliia Bykova, ${ }^{4}$ Markus Weigand, ${ }^{4}$ Mateusz Zelent ${ }^{2}$ Eberhard J. Goering, ${ }^{4}$ Gisela Schütz ${ }^{4}$ \\ Gianluca Gubbiotti, ${ }^{5}$ Maciej Krawczyk, ${ }^{2}$ Feliks Stobiecki, ${ }^{1}$ Janusz Dubowik, ${ }^{1}$ and Joachim Gräfe ${ }^{4}$ \\ ${ }^{1}$ Institute of Molecular Physics, Polish Academy of Sciences, Poznań, Poland \\ ${ }^{2}$ Faculty of Physics, Adam Mickiewicz University, Poznan, Poland \\ ${ }^{3}$ Institute of Physics, University of Greifswald, Greifswald, Germany \\ ${ }^{4}$ Max Planck Institute for Intelligent Systems, Stuttgart, Germany \\ ${ }^{5}$ Istituto Officina dei Materiali del Consiglio Nazionale delle Ricerche (IOM-CNR), Perugia I-06123, Italy
}

(Received 18 October 2018; revised manuscript received 26 March 2019; published 1 May 2019)

\begin{abstract}
Magnonic crystals are systems that can be used to design and tune the dynamic properties of magnetization. Here, we focus on one-dimensional Fibonacci magnonic quasicrystals. We confirm the existence of collective spin waves propagating through the structure as well as dispersionless modes; the reprogammability of the resonance frequencies, dependent on the magnetization order; and dynamic spin-wave interactions. With the fundamental understanding of these properties, we lay a foundation for the scalable and advanced design of spin-wave band structures for spintronic, microwave, and magnonic applications.
\end{abstract}

DOI: 10.1103/PhysRevApplied.11.054003

\section{INTRODUCTION}

In magnonic crystals (MCs), the band structure of allowed and forbidden frequencies for spin waves (SWs) can be tuned on demand by using an external magnetic field. This unique feature offers fine tuning and reprogrammability [1-4], which are desirable for potential applications [5] but are not easily obtained in photonic or plasmonic crystals [6]. Magnonic quasicrystals, which exhibit long-range order but no periodicity, offer possibilities beyond MC, such as fractal characteristics [7] and a larger number of forbidden and allowed magnonic bands, which are found in the linear regime (whereas in the case of MCs, fractality of power-frequency spectra can be found in the nonlinear regime [8]). Moreover, in quasicrystals, excitations can be localized in different regions of the structure, thus allowing for advanced tailoring of the properties of SWs [9].

Theoretical studies of magnonic Fibonacci quasicrystals are focused on multilayers [10-13] and, more recently, arrays of bicomponent nanowires [7,14,15]. Other works concern two-dimensional (2D) systems such as Penrose structures [16]. All these studies show the complexity and

\footnotetext{
*flisiecki@ifmpan.poznan.pl
}

Published by the American Physical Society under the terms of the Creative Commons Attribution 4.0 International license. Further distribution of this work must maintain attribution to the author(s) and the published article's title, journal citation, and DOI. self-similarity of the spectra. Experimental investigations of magnonic quasicrystals comprised of grooves arranged in a Fibonacci sequence etched in the micrometer-thick YIG film [17] show the influence of aperiodicity on the band structure. Other studies concern 2D systems of interconnected networks of permalloy $\left(\mathrm{Py}, \mathrm{Ni}_{80} \mathrm{Fe}_{20}\right.$ ) nanobars on Penrose P2 and P3 and Ammann quasicrystal lattices [18-21], as well as antidots arranged in octagonal lattices in Py [22]. However, there have been no investigations on magnonic quasicrystals, which would show the reprogrammability of the system and demonstrate how the properties of propagative SWs depend on the magnetic configuration, despite the great utility of this feature in magnonic systems. Other interesting systems are artificial nanostructures, in which magnetostatic interaction between properly ordered bistable magnetic grains can be designed to obtain magnetic frustration at the ground state [23-25] in, e.g., spin-ice systems. However, SW guiding has not yet been demonstrated in such structures.

In Ref. [26], we showed the possibility of effective SW propagation in quasiperiodic MCs, consisting of dipolarly coupled Py nanowire (NW) arrays and the opening of additional mini band gaps. In the present work, we exploit dynamic dipolar coupling in similar systems. In particular, we address the possibility of tuning the SW spectra of the magnonic quasicrystals in three ways: (i) by changing of the external magnetic field magnitude, allowing for continuous adjustment of the band and mini-band-gap 
frequencies; (ii) by switching of the magnetization configuration between parallel (ferromagnetic order, FO) and antiparallel (antiferromagnetic order, AFO), to demonstrate reprogrammability of the spectra; and (iii) by changing the dimensions of the magnetic elements, to show the scalability of the dynamical properties in an aperiodic system.

\section{METHODS}

The investigated system is sketched in Fig. 1. Py NWs (thickness $d=30 \mathrm{~nm}$, length $L=10 \mu \mathrm{m}$ ) are patterned by electron-beam lithography on silicon substrates. Two widths for narrow $W_{N}$ and wide $W_{W}=2 W_{N}$ stripes are chosen to be 350 and $700 \mathrm{~nm}$, and 700 and $1400 \mathrm{~nm}$, respectively. Dipolarly coupled NWs are separated by gaps $W_{G}=100 \mathrm{~nm}$ [27]. The quasiperiodic arrangement of the NWs is chosen according to the Fibonacci inflation rule, where a structure of a higher order $n$ is created as a concatenation of two previous structures: $S_{n}=S_{n-1}+S_{n-2}$ [14].

For SW excitation in vector network analyzerferromagnetic resonance (VNA-FMR) and scanning transmission x-ray microscopy (STXM) measurements, coplanar wave guides (CPWs) are deposited on top of the structures. These are aligned parallel to the NW axis. A static magnetic field $H_{\text {app }}$ is applied along the NW axis. Brillouin light scattering (BLS) experiments from thermally excited SWs are performed in the backscattering configuration by using a $200 \mathrm{~mW}$ solid-state laser operating at wavelength $\lambda=532 \mathrm{~nm}$. The sample is mounted on a goniometer, which allows us to choose a specified angle of incidence of light $(\theta)$, with an accuracy of $1^{\circ}$. Due to conservation of the in-plane momentum, by changing $\theta$ it is possible to select the magnitude of the in-plane component of the SW wave vector $[k=$ $(4 \pi / \lambda) \sin (\theta)]$ entering into the photon-magnon scattering process. To map the SW dispersion (frequency vs $k$ ), we vary $k$ in the range from 0 to $2 \times 10^{7} \mathrm{rad} / \mathrm{m} \mathrm{[28].} H_{\text {app }}$ is applied along the sample plane, parallel to the length of the nanowires (the $y$ direction) and perpendicular to the scattering plane (the $x-z$ plane) and the wave vector, in the so-called Damon-Eshbach (DE) configuration. The STXM measurements are carried out at the MAXYMUS end station at the Helmholtz-Zentrum Berlin, using the

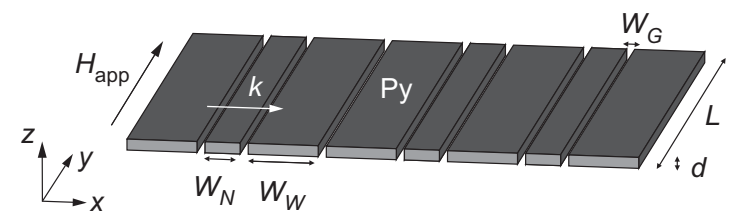

FIG. 1. A sketch of the $S_{6}$ Fibonacci structure consisting of Py nanowires of different widths separated by air gaps. The static magnetic field is applied along the nanowires.
Fe $L_{3}$ absorption edge for x-ray magnetic circular dichroism (XMCD) contrast $[29,30]$. To achieve time-resolved imaging, a sophisticated lock-in-like pump probe experiment using a custom FPGA for fast photon sorting and continuous-wave electrical radio-frequency (rf) excitation is used. A more detailed description of the STXM acquisition and analysis scheme can be found elsewhere [31]. The magneto-optic Kerr effect (MOKE) measurements are performed at room temperature, using a photoelastic modulator operating at $50 \mathrm{kHz}$ and lock-in amplification. Moreover, all these techniques are compared with numerical calculations.

To calculate the SW spectra, the Landau-Lifshitz equation is solved:

$$
\frac{\partial \mathbf{M}(\mathbf{r}, t)}{\partial t}=\mu_{0} \gamma \mathbf{M}(\mathbf{r}, t) \times \mathbf{H}_{\mathrm{eff}}(\mathbf{r}, t)
$$

where $t$ is the time, $\mathbf{r}$ is the position, and $\mathbf{M}$ is the magnetization. Damping is neglected in the calculations. The effective magnetic field $\mathbf{H}_{\text {eff }}(\mathbf{r}, t)$ is assumed to be the sum of the exchange, external, and dynamic demagnetizing fields. Assuming uniform excitation in the $y$ direction, Eq. (1) is linearized to obtain an eigenvalue problem in the frequency domain and real space, which is solved using a finite-element method (FEM) to obtain solutions in the form of monochromatic SWs with frequency $f$. For more details concerning the computational method, see Ref. [32]. In the numerical calculation, we adopt a saturation magnetization $M_{S}=0.76 \times 10^{6} \mathrm{~A} / \mathrm{m}$, an exchange constant $A=1.3 \times 10^{-11} \mathrm{~J} / \mathrm{m}$, and a gyromagnetic ratio $\gamma=1.76 \times 10^{11} \mathrm{rad} / \mathrm{sT}$ for Py.

\section{RESULTS}

To demonstrate the reprogrammability, scalability, and dynamic properties of Fibonacci MCs, three complementary techniques are used. The first one is VNA-FMR, to record the broadband resonance spectrum at different magnetic field values. The second technique is BLS spectroscopy, which allows mapping of the dispersion relation [33]. Finally, STXM is used to image the magnetization dynamics with spatial $(<20 \mathrm{~nm})$ and temporal $(<50$ ps) resolution. In these measurements, propagating and evanescent SWs are detected at frequencies inside of bands and band gaps, respectively. In addition, MOKE is used to measure the longitudinal hysteresis loops.

\section{A. The magneto-optic Kerr effect}

Figure 2 shows the hysteresis loops of the array of Fibonacci NWs for fields applied along the long (easy) axis of the NWs. As the applied field is increased from negative saturation, following the ascending branch of the $M-H$ loop, first a $100 \%$ remanence is visible and then, for arrays with NW widths of 700 and $1400 \mathrm{~nm}$ (350 and 700 


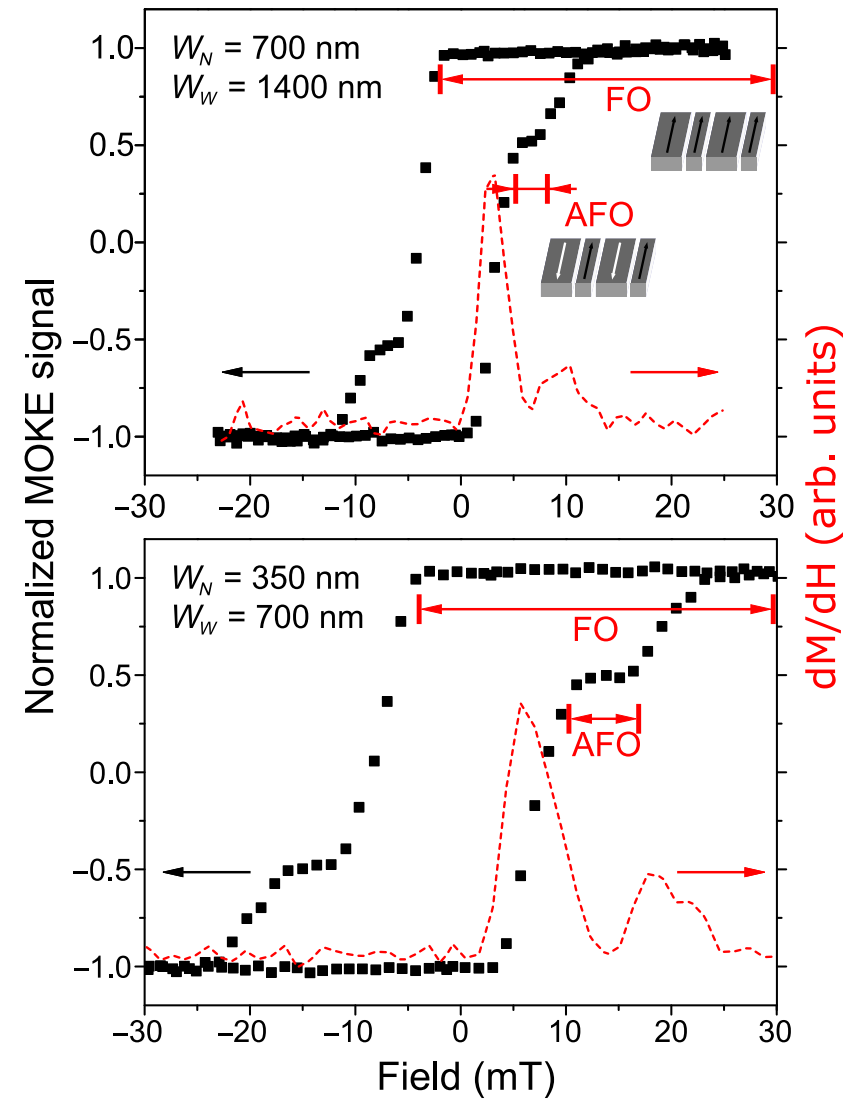

FIG. 2. The normalized MOKE hysteresis loop for the Fibonacci array with two values of stripe widths and the external field applied along the length of the NWs. The red dashed curve represents the first derivative of the lower branch of the loop with respect to the field. The arrows indicate the field range of the parallel (FO) and antiparallel (AFO) alignment of the wider and narrower NW magnetizations. Depending on the field history for an array consisting of 700- and 1400-nm or 350- and 700-nm NWs at $H=+5 \mathrm{mT}$ or $H=+12.5 \mathrm{mT}$, respectively, either an FO or an AFO magnetization orientation is present in the adjacent NWs.

$\mathrm{nm})$, a sharp drop in magnetization within the field range of $+1.5 \mathrm{mT}$ to $+4.9 \mathrm{mT}(+3 \mathrm{mT}$ to $+10 \mathrm{mT})$ is observed. This is followed by a quasistable plateau that spans to an external field of $+8 \mathrm{mT}(+16.5 \mathrm{mT})$. Beyond this field, a gradual increase in magnetization is seen up to a saturation field of $+12 \mathrm{mT}(+23 \mathrm{mT})$. This second jump is less pronounced, because intermediate states are stabilized by the dipolar interaction between adjacent NWs [34].

In general, the coercivity $H_{c}$ depends on the stripe width $W$ because of the shape anisotropy and scales as follows:

$$
H_{c}(W)=H_{c}^{0}+\frac{a}{W}
$$

where $a$ is a material parameter and $H_{c}^{0}$ is the coercivity of the unstructured material [35]. Because of this, a greater difference in the widths, especially for narrower
NWs, leads to a wider plateau with AFO in the hysteresis loop, which allows for a wider tuning range of the system [36,37].

For the same sample, we also plot (red curve) the differentiated half $M-H$ loop for fields applied from negative to positive saturation. The first peak corresponds to the low-field switching of the larger-width $\left(W_{W}\right)$ wire, while the second peak corresponds to the high-field switching of the smaller wire width $\left(W_{N}\right)$. The clear and distinct differences between the two peaks in the curve imply a region of antiparallel (AFO) alignment in the magnetization of neighboring wires in the array [36].

Based on these results, in the BLS and STXM measurements, field values of $H_{\text {app }}=+5 \mathrm{mT}$ or $+12.5 \mathrm{mT}$ are chosen for structures with NWs that are 700 and $1400 \mathrm{~nm}$ or 350 and $700 \mathrm{~nm}$ wide, respectively, to obtain the FO or AFO configuration, depending on the field history.

\section{B. VNA-FMR and numerical calculations}

In the absorption spectrum shown in Fig. 3, a strong signal from a collective excitation is observed. As expected, the resonance frequency can be continuously tuned by a

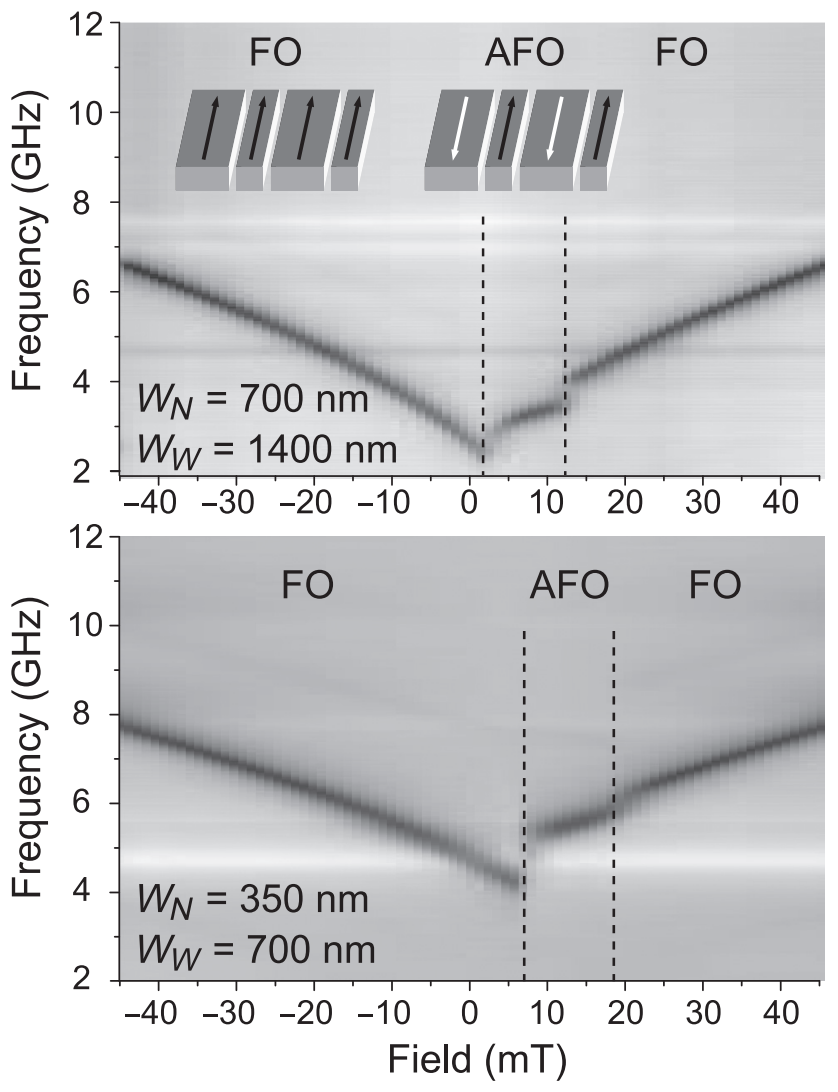

FIG. 3. The VNA-FMR spectra of the Fibonacci structures with two sets of wire widths. The field ranges in which ferromagnetic and antiferromagnetic orders are present after saturation in negative field values are separated by the vertical dashed lines and marked by FO and AFO, respectively. 
change of the magnetic field magnitude. When sweeping the field from negative to positive saturation, a strong asymmetry between negative and positive fields is observed. This is due to the magnetization reconfiguration upon reversal of the wide stripes [at 2 and $6.5 \mathrm{mT}$ for the (i) 700- and 1400-nm and (ii) 350- and 700-nm structures, respectively] into an AFO configuration and upon reversal of the narrow stripes (at 12 and $19 \mathrm{mT}$, respectively) back into a FO configuration [36]. These field values are in good agreement with the switching fields obtained from the MOKE measurements. The reconfiguration into an AFO state leads to a frequency shift of about $600 \mathrm{MHz}$ with respect to the FO state at the same field magnitude. This shows that the system's resonance frequencies can be reprogrammed by choosing an appropriate field sequence. Through tuning the dimensions of the NWs, the operational frequency and the degree of reprogrammability can be adjusted on demand.

To understand the observed frequency shift, numerical calculations of the SW band structure are conducted. Figure 4 shows the calculated dependence of frequency $f$ on the integrated density of states (IDOS) for the FO (black line) and AFO (red line) arrangements. Here, IDOS is defined as follows:

$$
\operatorname{IDOS}(f)=\int_{0}^{f} \sum_{i} \delta\left(f^{\prime}-f_{i}\right) d f^{\prime} \propto k(f),
$$

where $f$ is the frequency and $f_{i}$ is the eigenfrequency of the $i$ th SW mode. It can be shown (cf. the Appendix) that the IDOS $(f)$ for a one-dimensional (1D) system is proportional to the wave number $k(f)$. Therefore, the frequency vs IDOS relation reveals the dispersion relation (frequency vs wave number) in an extended-zone scheme. The distinctive jumps at the frequencies that are surrounded by the regions in which the dependence $f$ (IDOS) becomes flat can be identified as band gaps. Such jumps are clearly visible in Fig. 4. The band gaps for the FO and AFO states are marked by the violet and pink bars, respectively. Moreover, the calculated frequencies for the lowest IDOS (i.e., for $k \approx 0$ ) for both configurations are in good agreement with the VNA-FMR measurements (i.e., for $k=0$ ) shown in Fig. 3, indicating that the FMR for AFO is at a lower frequency due to the reduced effective magnetization [2]. These calculation results allow a simple optimization of the relevant parameters to tune the operating frequency of the Fibonacci structure in the AFO and FO configurations.

\section{Brillouin light scattering}

To go beyond the limitations of VNA-FMR and to observe the band structure of the magnonic quasicrystal, BLS spectroscopy is employed. In these measurements, the arrays with 350- and 700-nm-wide NWs are used. Measurements are performed in a field of $12.5 \mathrm{mT}$ after saturation from positive and negative field values for $\mathrm{FO}$ and

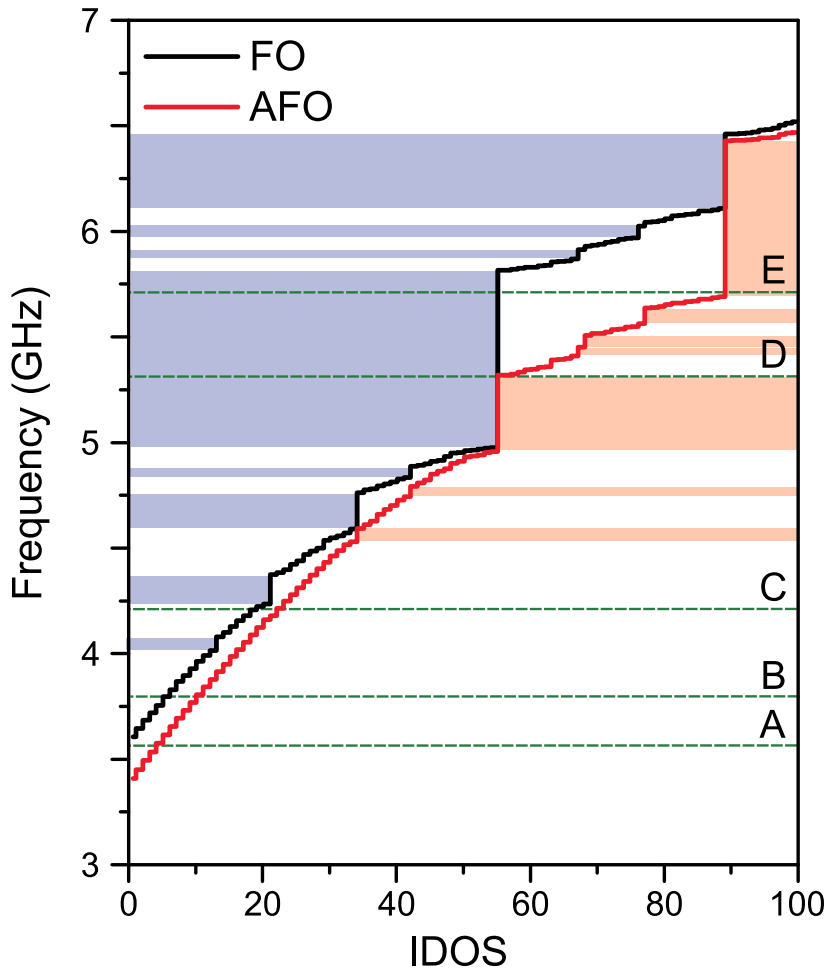

FIG. 4. The calculated band structure shown as frequency vs IDOS for structures with $W_{N}=700 \mathrm{~nm}$ and $W_{W}=1400 \mathrm{~nm}$, with ferromagnetic and antiferromagnetic order at $H_{\text {app }}=5$ and $-5 \mathrm{mT}$, respectively. The violet (pink) areas represent band gaps for FO (AFO). The dashed green lines mark the frequencies for which the STXM measurements are carried out.

AFO, respectively. Figure 5(b) shows the measured BLS spectra at $k=0.41 \times 10^{7} \mathrm{rad} / \mathrm{m}$ for FO and AFO. Both spectra exhibit several narrow and well-resolved peaks. In particular, a doublet of peaks is visible in the lowest frequency range of the spectra (below $9.0 \mathrm{GHz}$ ). Their frequency difference depends on the magnetization state. At the same time, a doublet of peaks is visible at a higher frequency (14.5 and $15.4 \mathrm{GHz}$ ) for the AFO state, while only one peak $(15.4 \mathrm{GHz})$ is observed for the FO. These are perpendicular standing SWs (PSSWs) and the doublet for AFO is caused by a different internal magnetic field of the narrow and wide NWs [38].

Figure 5(a) presents the measured dependence of the frequency on the wave vector $k$. In both magnetization configurations, the SW spectra are characterized by a set of discrete peaks, where only the lowest frequency mode exhibits a sizable dispersion. This is a clear indication that the Fibonacci array supports the propagation of longwavelength collective SWs in the FO and the AFO configurations. When $k$ is increased, other modes appear within a limited wave-vector range and are characterized by almost constant frequency values. These results are different from observations in periodic NW arrays of alternating width, where the modes follow Bloch's theorem with continuous 


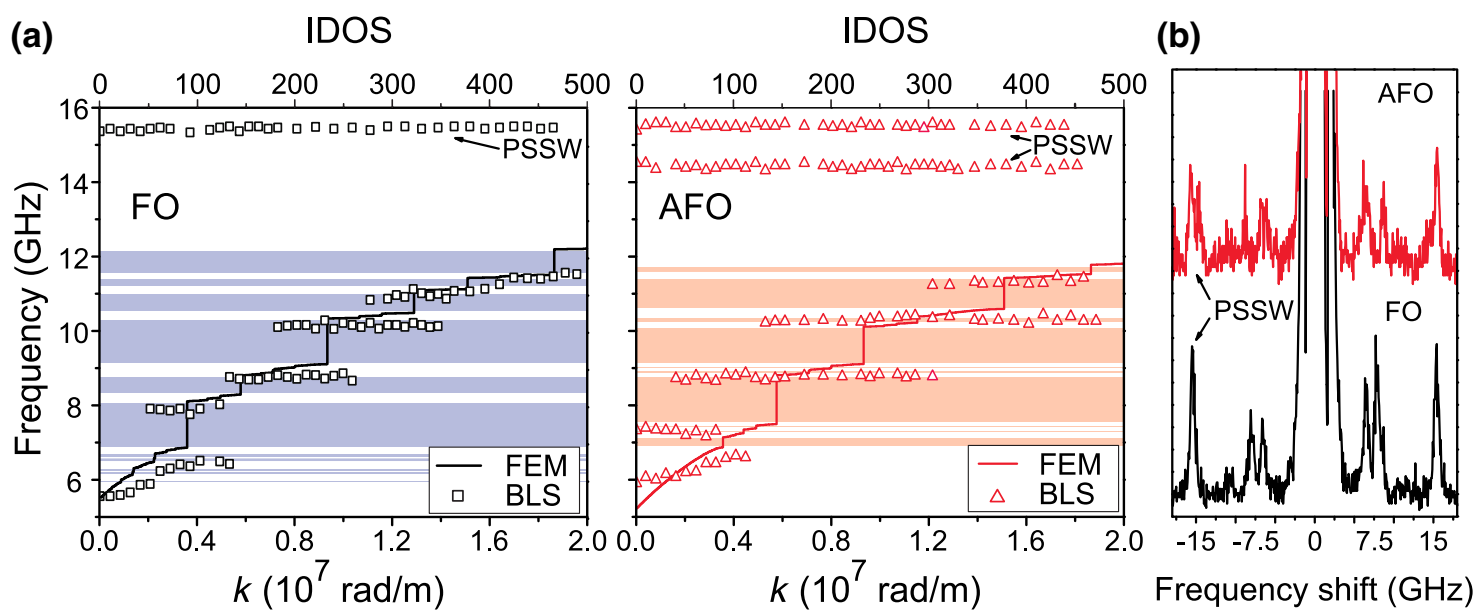

FIG. 5. (a) Experimental SW frequencies from BLS data vs wave vector $k$ compared with the band structure obtained from numerical calculations using the finite-element method (FEM, solid lines) shown as frequency vs IDOS for $H_{\text {app }}=+12.5 \mathrm{mT}$ for structures with $W_{N}=350 \mathrm{~nm}$ and $W_{W}=700 \mathrm{~nm}$ in ferromagnetic and antiferromagnetic order. The violet (pink) regions indicate the magnonic band gaps for FO (AFO) from the numerical calculations. (b) Typical experimental BLS spectra measured for $k=0.41 \times 10^{7} \mathrm{rad} / \mathrm{m}$.

dispersion and periodic frequency oscillation [39]. Apart from the previously discussed resonance frequency shift, other differences in the SW spectra between FO and AFO are also observed. The most significant difference is in the position of the second band. Just as in the structures with 700- and 1400-nm-wide NWs when the magnetization configuration changes from FO to AFO, there is a frequency downshift, which is not only wider than the resonance linewidth, but even wider than the width of the whole band, and thus could be used to control the SW transmission.

Furthermore, in Fig. 5(a), the BLS dispersion relation is compared to the computed frequencies of the SW excitations. It is important to note that these plots do not show a direct relation between IDOS and the $k$ vector but merely serve as a comparison of the allowed and forbidden frequency ranges. Very good agreement with the experimental data for the allowed bands and the band gaps in FO and AFO is found, justifying our analysis. Interestingly, apart from the shift to lower frequencies, such properties have already been found in the calculation results shown in Fig. 4, for a structure composed of NWs that have a doubled width. This shows that the properties of the Fibonacci magnonic quasicrystal are preserved regardless of its scale.

\section{X-ray microscopy}

Finally, to elucidate the microscopic behavior of the observed modes, the possibility of the control of their propagation hinted at by the BLS results, and their localization within the Fibonacci structures, the STXM measurements are conducted and interpreted in the framework of the numerical calculations. For structures with 700- and 1400nm-wide NWs, selected images of SWs excited by CPWs in $\mathrm{FO}$ and $\mathrm{AFO}$ for different excitation frequencies are presented in Fig. 6. The images are marked with the same letters A-E as in the numerical calculations in Fig. 4. The images are taken at $5 \mathrm{mT}$ and $-5 \mathrm{mT}$ for $\mathrm{FO}$ and AFO, respectively. The amplitude and phase are encoded as brightness and color, respectively.

On the right side of each image, the experimental excitation profiles (blue bars) of the out-of-plane magnetization component $m_{z}$ are plotted for an arbitrary time slice $t_{0}$ and compared with calculated profiles for propagating SWs (red lines). To match the calculation to experimental results, an exponential SW decay is taken into account. In general, very good agreement between the experimental and calculated excitation profiles is obtained for all modes - with the exception of mode D in AFO. It is noteworthy that additional SWs propagating across the NW in a backward-volume (BV) orientation are generally visible in our measurements [40]. For mode D in AFO, they interfere with SWs propagating across the array in such a way that a comparison with the calculations is difficult. Here, we focus on SWs propagating in DE geometry; thus, the interpretation of these orthogonal modes goes beyond the scope of the paper.

For $3.6 \mathrm{GHz}$ (marked as $\mathrm{A}$ ) in $\mathrm{FO}$, no $\mathrm{SW}$ propagation across the structure is observed, while there is an excited mode visible for AFO. Thus, frequency A for AFO lies in an allowed band and corresponds to the bottom part of the spectrum, while for FO this frequency is just below the first allowed band. This explains why no excitation for $\mathrm{FO}$ has been detected. For $3.8 \mathrm{GHz}$ (mode B) and $4.2 \mathrm{GHz}$ (mode $\mathrm{C}$ ), strongly excited SWs are seen in both configurations. For higher frequencies, as expected, shorter SWs are excited, which can easily be seen by the phase variation over a shorter distance. Modes B and C lie in the 


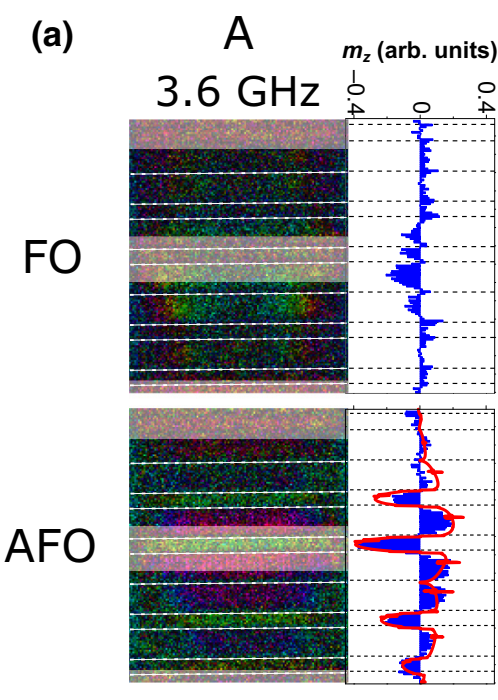

D
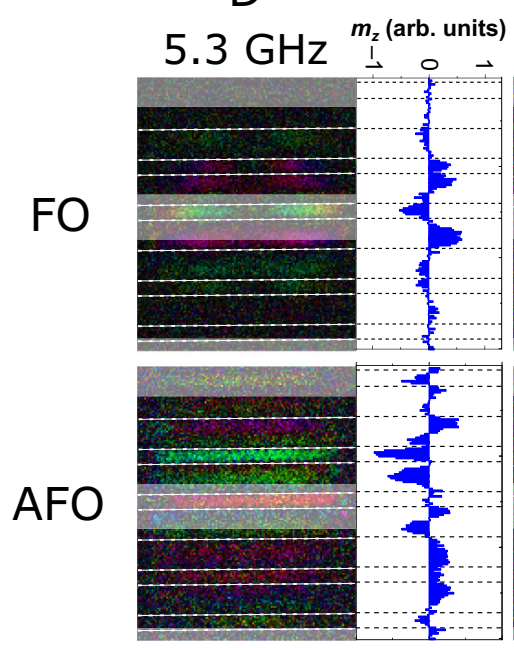

$\mathrm{B}$
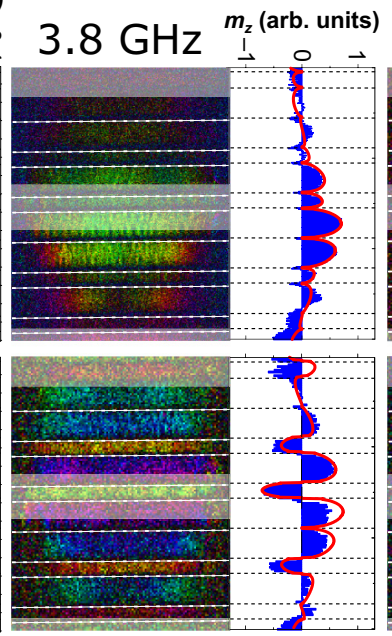

$\mathrm{E}$

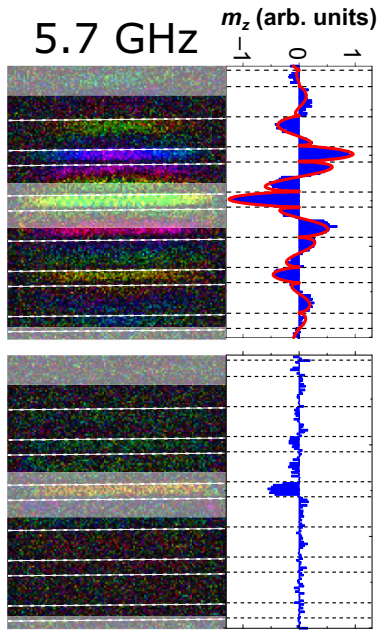

$\mathrm{C}$
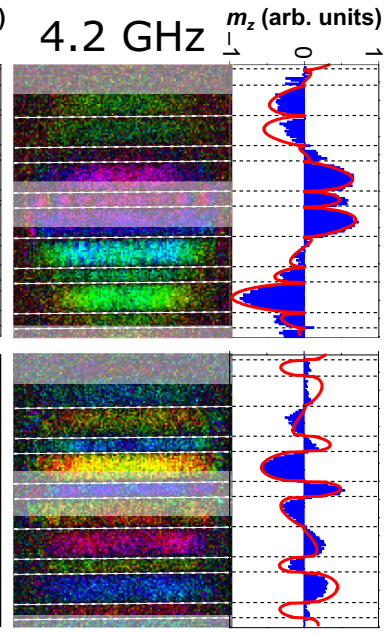

(b)
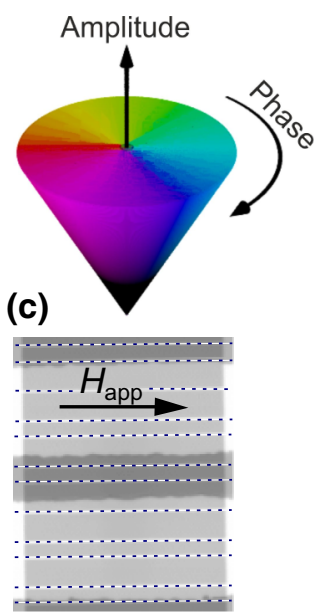

FIG. 6. (a) Amplitude-phase images from STXM measurements for selected excitation frequencies at $5 \mathrm{mT}$ and $-5 \mathrm{mT}$ for FO and AFO, respectively, for structures with $W_{N}=700 \mathrm{~nm}$ and $W_{W}=1400 \mathrm{~nm}$. The position of the CPW is marked with transparent gray rectangles and the gaps between the stripes with the dashed white lines. On the right side of each STXM image, experimental profiles of the excitations (blue bars) are plotted and compared with calculated ones (red solid lines). (b) The color codes for the SW amplitude (brightness) and phase (color). (c) A static image of the Fibonacci structure (light gray) with the visible parts of the CPW (dark gray) near the center of the image (the signal line) and at the top and bottom edges of the image (the ground lines).

first band, which explains the strong SW excitation at these frequencies.

A different situation is found at $5.3 \mathrm{GHz}$ (marked as D). A SW mode that propagates across the array with AFO is observed, while there is no SW mode for FO. For AFO, frequency $\mathrm{D}$ is close to the second band and therefore propagating SWs are visible; however, for FO it lies in the band gap between the first and the second band. This is why no propagating SWs are observed along the structure. On the other hand, at $5.7 \mathrm{GHz}$ the opposite situation is observed (marked as E). For FO, strong propagating SWs are again detected along the whole array, while for AFO, only weak forced excitation under the signal line is visible. For FO, frequency $\mathrm{E}$ is close to the second band, which explains the strong excitation. However, for AFO it lies in the band gap and SW propagation is suppressed. This confirms the possibility of controlling the SW transmission utilizing the second band, hinted at by the BLS results for a structure with narrower NWs.

It is worth noting that the influence of the quasiperiodicity is not clearly visible in the modes shown in Fig. 6, where we mainly focus on the issue of reprogrammability of the Fibonacci quasicrystal. This kind of irregularity of the modes and localization, characteristic for the quasiperiodic structures, would be expected to be more pronounced for the modes from higher bands, which we do not show 
here. Nevertheless, the influence of quasiperiodicity on the lower bands can be seen in the band structure as mini band gaps, which originate from a dense spectrum of diffraction peaks in reciprocal space. This is the subject of analysis in another work [26].

In all of the images of SW modes for FO, there is a gradual variation of the phase along the propagation direction, confirming the propagating character of SWs. This is also the case for AFO, although there are $180^{\circ}$ phase jumps between the wide and narrow stripes resulting from the opposite directions of the magnetization precession in these stripes. This indicates an opposing orientation of the out-of-plane magnetization oscillations in wide and narrow NWs [2]. However, it does not disrupt the propagation of SWs. Those results show that the static magnetization configuration [41] and the dynamic coupling (investigated in this paper) are determined by different magnetization components: along the NW axis and perpendicular to that direction, respectively. This provides a hint for the design of artificial spin systems with various stable magnetization configurations that allow SW propagation.

\section{SUMMARY}

In summary, we investigate, experimentally and numerically, SW dynamics in 1D magnonic quasicrystals composed of Py NWs of two different widths, arranged in a Fibonacci sequence, to demonstrate the power of these systems to precisely tune the SW spectra. The combination of VNA-FMR, BLS spectroscopy, and STXM imaging allows us to confirm experimentally the SW dispersion relation in magnonic quasicrystals and to develop a precise understanding of the individual modes.

We find that the change from a FO to an AFO magnetization configuration of the NWs induces strong variations in the SW dynamics. Both the frequency of the collective modes and the positions of the magnonic bands are shifted between the two configurations. These can easily be tuned by an appropriate choice of the NW widths, even beyond the 350-1400-nm range demonstrated here.

The propagation of SWs across all wires in AFO indicates an additional magnetic coupling mechanism in these artificial spin structures. Apart from the magnetostatic interaction, we find a dynamic coupling of SWs that leads to the opposing sign of the magnetization oscillation in narrow and wide stripes and does not hinder the propagation of SWs. This coupling is a tool for the design of artificial spin structures and possible frustrated systems based on bistable NWs, in the $2 \mathrm{D}$ plane too.

The observed properties of the Fibonacci structures hint at their applicability toward magnonic devices for data processing and wave-based computing. Beyond offering more precise control of SW spectra than magnonic crystals, they are conveniently reprogrammable. Their use for a fieldreprogrammable SW filter, where the SW transmission of a given SW frequency could be controlled by changing between the FO and AFO magnetization configurations, can easily be envisaged. Another use of such a reconfiguration would be a phase shifter. In that case, the dynamic coupling of adjacent stripes would lead to a $180^{\circ}$ shift in phase for the magnetization reconfiguration.

\section{ACKNOWLEDGMENTS}

The study has received funding from the European Union's Horizon 2020 research and innovation programme under Marie Skodowska-Curie GA No. 644348 (MagIC), from the Polish Ministry of Science and Higher Education, granted for the realization of an international cofinanced project (W28/H2020/2017), from the National Science Centre of Poland under Grants No. UMO-2016/21/B/ST3/00452 and No. UMO2017/24/T/ST3/00173, and from the Adam Mickiewicz University Foundation. We thank the Helmholtz-Zentrum Berlin for the allocation of synchrotron radiation beam time. J.W.K. would like to acknowledge the support of the Foundation of Alfried Krupp Kolleg Greifswald.

F.L. and J.R. contributed equally to this work.

\section{APPENDIX: THE RELATION BETWEEN IDOS AND WAVE NUMBER $K$ IN 1D SYSTEMS}

In a 1D system of size $L$, the number of states per unit interval in the reciprocal space of the wave number is equal to the following:

$$
n(k)=\frac{L}{2 \pi} .
$$

When the system is large $(L \gg 2 \pi / k)$, the number of states in an infinitesimally small interval of reciprocal space, $n(k) d k$, can be expressed by the density of states (DOS), $\operatorname{DOS}(f):=(d / d f) n(f)$, in the following form:

$$
n(k) d k=\operatorname{DOS}(f(k)) d f .
$$

Equation (A2) allows us to relate the DOS to the derivative $d k / d f$ :

$$
\operatorname{DOS}(f)=\frac{L}{2 \pi}\left|\frac{d k}{d f}\right|
$$

The integrated density of states IDOS $(f)$ is a number of states of the frequencies below the frequency $f$ :

$$
\operatorname{IDOS}(f)=\int_{0}^{f} \operatorname{DOS}\left(f^{\prime}\right) d f^{\prime}=\frac{L}{2 \pi}|k(f)| .
$$

For the (quasi)periodic system, the dispersion relation $k \leftrightarrow f$ in Eq. (A4) must be taken as an injective function, i.e., it has to be considered in the extended-zone scheme 
without folding by reciprocal lattice vectors: $k \rightarrow k-G$. The formula in Eq. (A4) relates the IDOS to the wave vector $k$.

The $\operatorname{IDOS}(f)$ is a nondecreasing function of the frequency. In the (quasi)periodic system at the frequency gaps, $\operatorname{DOS}(f)=0$. In the frequency gaps, the IDOS is constant, whereas at the gap edges its slope $(d / d f)(\operatorname{IDOS}(f))=\operatorname{DOS}(f)$ diverges. This divergence is related to the presence of Van Hove singularities for the 1D system. For real systems, we have to include their finite sizes. The DOS $(f)$ is then a sequence of Dirac deltas and the IDOS $(f)$ steplike function. The wide steps of IDOS $(f)$ surrounded by the sequences of steps with rapidly decreasing widths are the signatures of the frequency gap in the $1 \mathrm{D}$ system.

[1] M. Krawczyk and D. Grundler, Review and prospects of magnonic crystals and devices with reprogrammable band structure, J. Phys.: Condens. Matter 26, 123202 (2014).

[2] Jesco Topp, Detlef Heitmann, Mikhail P. Kostylev, and Dirk Grundler, Making a Reconfigurable Artificial Crystal by Ordering Bistable Magnetic Nanowires, Phys. Rev. Lett. 104, 207205 (2010).

[3] G. Gubbiotti, X. Zhou, Z. Haghshenasfard, M. G. Cottam, and A. O. Adeyeye, Reprogrammable magnonic band structure of layered permalloy/Cu/permalloy nanowires, Phys. Rev. B 97, 134428 (2018).

[4] S. Tacchi, B. Botters, M. Madami, J. W. Kłos, M. L. Sokolovskyy, M. Krawczyk, G. Gubbiotti, G. Carlotti, A. O. Adeyeye, S. Neusser, and D. Grundler, Mode conversion from quantized to propagating spin waves in a rhombic antidot lattice supporting spin wave nanochannels, Phys. Rev. B 86, 014417 (2012).

[5] A. V. Chumak, V. I. Vasyuchka, A. A. Serga, and B. Hillebrands, Magnon spintronics, Nat. Phys. 11, 453 (2015).

[6] Nikolay I. Zheludev and Yuri S. Kivshar, From metamaterials to metadevices, Nat. Mater. 11, 917 (2012).

[7] J. Rychły, J. W. Kłos, M. Mruczkiewicz, and M. Krawczyk, Spin waves in one-dimensional bicomponent magnonic quasicrystals, Phys. Rev. B 92, 054414 (2015).

[8] Daniel Richardson, Boris A. Kalinikos, Lincoln D. Carr, and Mingzhong $\mathrm{Wu}$, Spontaneous Exact Spin-Wave Fractals in Magnonic Crystals, Phys. Rev. Lett. 121, 107204 (2018).

[9] C. H. Chen, R. Z. Qiu, C. H. Chang, and W. J. Hsueh, Strongly localized modes in one-dimensional defect-free magnonic quasicrystals, AIP Adv. 4, 087102 (2014).

[10] C. H. O. Costa, M. S. Vasconcelos, and E. L. Albuquerque, Partial band gaps in magnonic crystals, J. Appl. Phys. 109, $07 \mathrm{D} 319$ (2011).

[11] C. H. O. Costa and M. S. Vasconcelos, Band gaps and transmission spectra in generalized Fibonacci $(p, q)$ onedimensional magnonic quasicrystals, J. Phys.: Condens. Matter 25, 286002 (2013).

[12] I. P. Coelho, M. S. Vasconcelos, and C. G. Bezerra, Quasiperiodic magnonic superlattices with mirror symmetry, Solid State Commun. 150, 1760 (2010).
[13] I. P. Coelho, M. S. Vasconcelos, and C. G. Bezerra, Transmission fingerprints in quasiperiodic magnonic multilayers, J. Magn. Magn. Mater. 323, 3162 (2011).

[14] J. Rychły, J. W. Kłos, and M. Krawczyk, Spin wave damping in periodic and quasiperiodic magnonic structures, J. Phys. D: Appl. Phys. 49, 175001 (2016).

[15] B. Hussain, M. G. Cottam, and G. Baolai, Quasiperiodic spin waves in bi-component magnonic crystal arrays of nanowires, J. Phys. D: Appl. Phys. 52, 095001 (2018).

[16] J. Rychły, S. Mieszczak, and J. W. Kłos, Spin waves in planar quasicrystal of Penrose tiling, J. Magn. Magn. Mater. 450, 18 (2018).

[17] S. V. Grishin, E. N. Beginin, Yu. P. Sharaevskii, and S. A. Nikitov, Dissipative soliton generation in an active ring resonator based on magnonic quasicrystal with Fibonacci type structure, Appl. Phys. Lett. 103, 022408 (2013).

[18] V. S. Bhat, J. Sklenar, B. Farmer, J. Woods, J. T. Hastings, S. J. Lee, J. B. Ketterson, and L. E. De Long, Controlled Magnetic Reversal in Permalloy Films Patterned into Artificial Quasicrystals, Phys. Rev. Lett. 111, 077201 (2013).

[19] V. S. Bhat, J. Sklenar, B. Farmer, J. Woods, J. B. Ketterson, J. T. Hastings, and L. E. De Long, Ferromagnetic resonance study of eightfold artificial ferromagnetic quasicrystals, J. Appl. Phys. 115, 17C502 (2014).

[20] B. Farmer, V. S. Bhat, J. Sklenar, E. Teipel, J. Woods, J. B. Ketterson, J. T. Hastings, and L. E. De Long, Magnetic response of aperiodic wire networks based on Fibonacci distortions of square antidot lattices, J. Appl. Phys. 117, 17B714 (2015).

[21] V. S. Bhat and D. Grundler, Angle-dependent magnetization dynamics with mirror-symmetric excitations in artificial quasicrystalline nanomagnet lattices, Phys. Rev. B 98, 174408 (2018).

[22] Samiran Choudhury, Saswati Barman, Yoshi Chika Otani, and Anjan Barman, Efficient modulation of spin waves in two-dimensional octagonal magnonic crystal, ACS Nano 11, 8814 (2017).

[23] L. J. Heyderman and R. L. Stamps, Artificial ferroic systems: Novel functionality from structure, interactions and dynamics, J. Phys.: Condens. Matter 25, 363201 (2013).

[24] Joachim Gräfe, Felix Haering, Thomas Tietze, Patrick Audehm, Markus Weigand, Ulf Wiedwald, Paul Ziemann, Przemysaw Gawroski, Gisela Schütz, and Eberhard J. Goering, Perpendicular magnetisation from in-plane fields in nano-scaled antidot lattices, Nanotechnology 26, 225203 (2015).

[25] J. Dubowik, P. Kusświk, M. Matczak, W. Bednarski, F. Stobiecki, P. Aleshkevych, H. Szymczak, M. Kisielewski, and J. Kisielewski, Ferromagnetic resonance and resonance modes in kagome lattices: From an open to a closed kagome structure, Phys. Rev. B 93, 224423 (2016).

[26] F. Lisiecki, J. Rychły, P. Kuświk, H. Głowiński, J. W. Kłos, F. Groß, I. Bykova, M. Weigand, M. Zelent, E. Goering, G. Schütz, M. Krawczyk, F. Stobiecki, J. Dubowik, and J. Gräfe, Magnons in a Quasicrystal: Propagation, Localization and Extinction of Spin Waves in Fibonacci Structures, arXiv:1810.04931 [cond-mat.mes-hall] (2018).

[27] G. Gubbiotti, S. Tacchi, G. Carlotti, N. Singh, S. Goolaup, A. O. Adeyeye, and M. Kostylev, Collective spin modes in 
monodimensional magnonic crystals consisting of dipolarly coupled nanowires, Appl. Phys. Lett. 90, 092503 (2007).

[28] Marco Madami, Gianluca Gubbiotti, Silvia Tacchi, and Giovanni Carlotti, in Solid State Physics, edited by R. E. Camley and R. L. Stamps (Academic Press, Burlington, MA, 2012), Vol. 63, p. 79.

[29] D. Nolle, M. Weigand, P. Audehm, E. Goering, U. Wiesemann, C. Wolter, E. Nolle, and G. Schütz, Note: Unique characterization possibilities in the ultrahigh-vacuum scanning transmission x-ray microscope (UHV-STXM) "MAXYMUS" using a rotatable permanent magnetic field up to $0.22 \mathrm{~T}$, Rev. Sci. Instrum. 83, 046112 (2012).

[30] Joachim Gräfe, Markus Weigand, Nick Träger, Gisela Schütz, Eberhard J. Goering, Maxim Skripnik, Ulrich Nowak, Felix Haering, Paul Ziemann, and Ulf Wiedwald, Geometric control of the magnetization reversal in antidot lattices with perpendicular magnetic anisotropy, Phys. Rev. B 93, 104421 (2016).

[31] Felix Groß, Nick Träger, Johannes Förster, Markus Weigand, Gisela Schütz, and Joachim Gräfe, Nanoscale detection of spin wave deflection angles in permalloy, Appl. Phys. Lett. 114, 012406 (2019).

[32] M. Mruczkiewicz, M. Krawczyk, V. K. Sakharov, Yu. V. Khivintsev, Yu. A. Filimonov, and S. A. Nikitov, Standing spin waves in magnonic crystals, J. Appl. Phys. 113, 093908 (2013).

[33] S. Tacchi, G. Gubbiotti, M. Madami, and G. Carlotti, Brillouin light scattering studies of 2D magnonic crystals, J. Phys.: Condens. Matter 29, 073001 (2017).

[34] Felix Groß, Sven Erik Ilse, Gisela Schütz, Joachim Gräfe, and Eberhard Goering, Interpreting first-order rever- sal curves beyond the Preisach model: An experimental permalloy microarray investigation, Phys. Rev. B 99, 064401 (2019).

[35] Joachim Gräfe, Gisela Schütz, and Eberhard J. Goering, Coercivity scaling in antidot lattices in $\mathrm{Fe}, \mathrm{Ni}$, and $\mathrm{NiFe}$ thin films, J. Magn. Magn. Mater. 419, 517 (2016).

[36] S. Goolaup, A. O. Adeyeye, N. Singh, and G. Gubbiotti, Magnetization switching in alternating width nanowire arrays, Phys. Rev. B 75, 144430 (2007).

[37] J. Ding, M. Kostylev, and A. O. Adeyeye, Magnetic hysteresis of dynamic response of one-dimensional magnonic crystals consisting of homogenous and alternating width nanowires observed with broadband ferromagnetic resonance, Phys. Rev. B 84, 054425 (2011).

[38] J. Jorzick, S. O. Demokritov, B. Hillebrands, M. Bailleul, C. Fermon, K. Y. Guslienko, A. N. Slavin, D. V. Berkov, and N. L. Gorn, Spin Wave Wells in Nonellipsoidal Micrometer Size Magnetic Elements, Phys. Rev. Lett. 88, 047204 (2002).

[39] S. Tacchi, M. Madami, G. Gubbiotti, G. Carlotti, S. Goolaup, A. O. Adeyeye, N. Singh, and M. P. Kostylev, Analysis of collective spin-wave modes at different points within the hysteresis loop of a one-dimensional magnonic crystal comprising alternative-width nanostripes, Phys. Rev. B 82, 184408 (2010).

[40] See the Supplemental Material at http://link.aps.org/supple mental/10.1103/PhysRevApplied.11.054003 for the videos from the STXM measurements.

[41] K. Szulc, F. Lisiecki, A. Makarov, M. Zelent, P. Kuświk, H. Głowiński, J. W. Kłos, M. Münzenberg, R. Gieniusz, J. Dubowik, F. Stobiecki, and M. Krawczyk, Remagnetization in arrays of ferromagnetic nanostripes with periodic and quasiperiodic order, Phys. Rev. B 99, 064412 (2019). 